Bundesgesundheitsbl -

Gesundheitsforsch - Gesundheitsschutz

2001 · 44:491-497 @ Springer-Verlag 2001

Originalien und Übersichtsarbeiten

A. Nassauer • Robert Koch-Institut, Berlin

\title{
Umsetzung des Infektions- schutzgesetzes (IfSG) - Aufgaben für die Arbeitsmedizin?
}

\section{Zur Kooperation von Gesundheitsämtern und Betriebsärzten}

Konkretisierung der allgemeinen Vorschriften des IfSG (§1 Abs. 2 und § 3), die als Leitfaden des Gesetzes, Prävention durch Information und Aufklärung" und „Förderung der Eigenverantwortung von Einrichtungen und Betrieben" postulieren. Aufgrund der Schulgesetze, der Gesetze über den Öffentlichen Gesundheitsdienst (ÖGD) und der Regelungen in § 34 IfSG haben Gesundheitsämter „betriebsärztliche Aufgaben“ in Schulen und sonstigen Gemeinschaftseinrichtungen. Allerdings hat der Arbeitsaufwand für den ÖGD aufgrund der alle zwei Jahre zu wiederholenden Belehrungen für das Personal der Einrichtungen weiter zugenommen. Es bietet sich daher an, dass private Ärzte für Arbeitsmedizin mit dem ÖGD kooperieren und Schulungsaufgaben übernehmen. Die Erstbelehrung für Beschäftigte in Lebensmit- telbetrieben erfolgt gem. $§ 43$ Abs. 1 IfSG in mündlicher und schriftlicher Form durch das Gesundheitsamt. Die Wiederholungsbelehrungen haben danach in den Betrieben jährlich zu erfolgen. Sie sind zwar Sache des Unternehmers, aber wegen der medizinischen Inhalte sollte auch hierfür wieder ärztlicher Sachverstand herangezogen werden. Gerade in diesem Bereich sind Absprachen und gegenseitige Unterstützung von ÖGD und Betriebsmedizin erforderlich, damit das vom IfSG geforderte Ziel (Aufklärung und Eigenverantwortung) auch tatsächlich erreicht wird.

\section{Schlüsselwörter}

Arbeitsmedizin - Betriebsarzt ·

Bundes-Seuchengesetz - Infektionsschutzgesetz - Gemeinschaftseinrichtungen . Lebensmittelbetriebe

nungsgesetzes. Diese Bezeichnung veranlasst juristisch ungeübte Leser zu der Annahme, dass der Gesetzgeber das BSeuchG in einigen Passagen zwar geändert, aber im Wesentlichen alles beim Alten gelassen habe. Wäre dies das Anliegen gewesen, hätte der Bundestag ein Artikelgesetz zum BSeuchG beraten und verabschiedet. Richtig ist, dass bewährte Vorschriften des BSeuchG übernommen wurden (z. B. mehrere Vorschriften zur Verhütung und zur Bekämpfung übertragbarer Krankheiten). Aber schon beim Studium des Inhaltsverzeichnisses fällt auf, dass

- die allgemeinen Vorschriften,

- das Meldewesen,

- die Vorschriften für die Krankenhaushygiene,

- diejenigen für Schulen und sonstige Gemeinschaftsreinrichtungen und

- diejenigen für Beschäftigte in Lebensmittelbetrieben

as Bundes-Seuchengesetz (BSeuchG) war 40 Jahre geltendes Recht, und Verwaltungspraxis, Rechtsprechung, wissenschaftliche Veröffentlichungen und die Kommentatoren haben im Laufe der Zeit Beiträge zur Umsetzung dieses Gesetzes geleistet. Dies ist regelmäßig der Weg, wie die gerade von juristischen Laien geforderte Rechtssicherheit geschaffen wird.

Das seit dem 1.1.2001 geltende Infektionsschutzgesetz (IfSG) ist als der Art. 1 der umfangreichste und bedeutendste Teil des Seuchenrechtsneuord- grundlegend neu gestaltet worden sind. Damit ist die Erwartung verbunden, dass Infektionsprävention und die Bekämpfung übertragbarer Krankheiten auch tatsächlich verbessert werden.

Folglich wurde an das Robert KochInstitut mehrfach die Frage gerichtet, ob man davon ausgehen könne, dass sich

Dr. Alfred Nassauer Robert Koch-Institut, Postfach 650280, 13302 Berlin 
Bundesgesundheitsbl -

Gesundheitsforsch - Gesundheitsschutz

2001 · 44:491-497 @ Springer-Verlag 2001

\section{A. Nassauer}

Implementation of the Infection Protection Act (IfSG) - Tasks for occupational medicine. Cooperation between local health authorities and company physicians

\section{Abstract}

As announced in the Federal Law Gazette (July 2000), the Infection Protection Act (IfSG) replaced the Federal Law for Control of Infectious Diseases (BSeuchG) on the first of January, 2001, ending its reign for the past forty years. Incorporating some of the tried and tested rules from the BSeuchG, the Infection Protection Act contains new rules on case reporting procedures, hospital hygiene, communal establishments and food handling enterprises, which will gradually be implemented through administrative channels, jurisdiction, professional editorials and academic publications. In place of requiring physical examinations, $x$-rays and stool specimens from employees in communal establishments and food handling enterprises according to the BSeuchG, the Infection Protection Act holds informative talks with workers in these fields on infectious diseases, on favourable conditions for spreading the disease, and on how agents contaminate certain products in food handling enterprises that can cause consumers to become ill. These informative talks with both employees and visitors in communal establishments and with employees in food handling enterprises (see further $\S \S 34 / 35$ and 42/43 IfSG) are based on the general rules of the Infection Protection Act (§ 1 Para. 2 and § 3), which adhere to the guidelines found in the leaflets "Prevention by information and education" and "Fostering personal responsibility in communal establishments and enterprises."On the basis of school legislation, public health law and the rules stated in $\S 34$ of the Infection Protection Act, community health services must fulfil the duties of a occupational health physician in schools and other communal institutions. Because of the need to repeat training sessions for employees in these institutions every two years, the work load of public health officials has increased sharply. Training sessions are oral and entail handing out leaflets. Depending on the degree of expertise, these sessions can be quite time consuming. However, quality must not be sacrificed in order to ease the work load. Consequently, general practitio-

Originalien und Übersichtsarbeiten

ners in occupational health are expected to cooperate with public health services and take over these training sessions. The initial informative talk with the employees in food handling enterprises is, in accordance with $\S 43$ Para 1 of The Infection Protection Act, both oral and written and is administered by the community health service. Further instructional sessions on diarrhoea, typhus, hepatitis $A$, suppurating skin diseases, among others (see $\S 42$ IfSG) should be held annually in the respective enterprises. While the employer is responsible for holding these meetings, their medicinal nature requires the competence of a doctor. In 1999, Charlottenburg, a district in Berlin, issued 8,650 certificates; Frankfurt am Main issued around 6,600 the same year. These examples show clearly how much time is required for the initial and following instructions. In order to reach the goal of having employees who are both informed and responsible, a mutual understanding and good communications between the public health service and occupational health are of utmost importance. Dispensing of the required stool specimen in accordance with $\S \S 17 / 18$ of the BSeuch $G$ has been heavily criticised, for fear that an undetected carrier could endanger the purity of a product. However, the prevalence of positive stool specimen ranged between 0,3 and 0,7 percent in the $90 \mathrm{~s}$ and any relationship between the examination and infectious epidemiological insight of infections due to food contamination could not be proven. Surely, this shows that the best way to prevent infections is by informing and instructing people about the dangers.

\section{Keywords}

Federal Law for Control of Infectious Diseases - Infection Protection Act . Communal institutions · Food handling enterprises - occupational medicine - Local health authorities - Company physicians

die „Meldemoral“ der Ärzte aufgrund der Neuregelung im IfSG verbessern werde. Eine Änderung der Rechtslage (Verkündigung des IfSG im Bundesgesetzblatt im Juli 2000) allein hat zunächst keine merklichen Auswirkungen. Erst wenn Behörden oder auch private Adressaten durch oder aufgrund eines neuen Gesetzes zu entscheiden oder sonst zu handeln haben, werden die
Auswirkungen neuer Regelungen spürbar und nach und nach bekannt.

Da das RKI an mehreren Stellen im Gesetz explizit genannt und seine Aufgaben in $\$ 4$ IfSG beschrieben werden, liegt es nahe, dass auch aus der Ärzteschaft aktuell zahlreiche Anfragen an diese Bundesoberbehörde gerichtet werden. Ein niedergelassener Mikrobiologe fragte z. B. im Januar 2001 bei der Pressestelle des Institutes an, warum man ihm (als besonders betroffenen Facharzt) den Gesetzestext nicht geschickt habe. Die Antwort enthielt die Internetadresse, unter der das IfSG und alle anderen Dokumente zum IfSG aus dem RKI eingesehen werden können: www.rki.de/INFEKT/IFSG/IFSG. HTM.

Die beiden Beispiele zeigen neben einer gewissen Erwartungshaltung auch einen großen Informationsbedarf der Fachöffentlichkeit, der durch die Multiplikatoren nur schrittweise befriedigt werden kann. Augenblicklich wichtigste Quelle ist sicherlich das Themenheft des Bundesgesundheitsblattes aus dem November 2000.

Mit diesem Beitrag soll eine weitere Facette der gesetzlichen Neuregelungen beleuchtet werden: Im BSeuchG waren Untersuchungspflichten für Beschäftigte in Gemeinschaftseinrichtungen und Lebensmittelbetrieben normiert, die nicht ins IfSG übernommen wurden. An ihre Stelle sind Belehrungen getreten, die insgesamt zeitaufwendiger sein dürften. Außerdem wurden Einrichtungen und Unternehmen Wiederholungsbelehrungen auferlegt; dabei hat das $\mathrm{Ge}$ setz offen gelassen, durch welche fachlich qualifizierten Personen gerade diese Wiederholungsbelehrungen durchgeführt werden sollten.

Wie zwischen Gesundheitsämtern, die auf der kommunalen Ebene mannigfach arbeitsmedizinische Aufgaben in Behörden und Einrichtungen der Landkreise und kreisfreien Städte wahrnehmen und privaten bzw. niedergelassenen Betriebsärzten eine Kooperation aber auch eine Aufgabenteilung einvernehmlich etabliert werden kann, soll in diesem Beitrag dargestellt werden.

\section{Infektionsschutz und Arbeitsmedizin}

Allein durch den Regelungsgehalt der Biostoffverordnung (BioStoffV) wird deutlich, dass Infektionsschutz und me- 
dizinischer Arbeitsschutz untrennbar miteinander verbundene Rechtsbereiche sind [1]. Zweck des IfSG ist, den Einzelnen wie die Allgemeinheit vor übertragbaren Krankheiten zu schützen $(\$ 1$ Abs. 1 und $\$ 3$ IfSG); die BioStoffV enthält Regelungen zum Schutz von Beschäftigten vor biologischen Arbeitsstoffen (zu denen auch Krankheitserreger gehören, $\$ 2$ BioStoffV) am Arbeitsplatz. Sie verfolgt damit innerhalb ihres Anwendungsbereichs die gleiche Zielrichtung wie das IfSG. Da jedoch die Rechtsbegriffe medizinischer Arbeitsschutz, Arbeitsmedizin, Betriebsarzt oder verwandte Termini im IfSG nicht zu finden sind, fehlt es für Betriebsärzte an Rechtssicherheit, in welchen Bereichen des Infektionsschutzgesetzes ihnen Aufgaben übertragen sind oder durch sie kompetent erfüllt werden können.

\section{Prävention durch Information und Aufklärung}

$\$ 3$ Arbeitssicherheitsgesetz beschreibt die Aufgaben von Betriebsärzten und führt in Abs. 1 Nr. 4 aus: „(Sie haben) darauf hinzuwirken, dass sich alle im Betrieb Beschäftigten den Anforderungen des Arbeitsschutzes entsprechend verhalten, insbesondere sie über Unfallund Gesundheitsgefahren, denen sie bei der Arbeit ausgesetzt sind, sowie über die Einwirkungen und Maßnahmen zur Abwendung dieser Gefahren zu belehren und.... bei der Schulung.... des medizinischen Hilfspersonals mitzuwirken.“ Auch diese Vorschrift ist eindeutig eine Beschreibung von Primärprävention im Arbeitsschutz und setzt auf „belehren“ und „schulen“. Nicht verschwiegen werden soll, dass $₫ 3$ Nr. 1 der Vorschrift die Beratung des Arbeitgebers, Nr. 2 die arbeitsmedizinischen Vorsorgeuntersuchungen und Nr. 3 die Beobachtung der Einhaltung des Arbeitsschutzes (z. B.im Rahmen von Begehungen) als weitere Aufgaben beschreiben; damit prägen sowohl Kontrolle als auch Beratungselemente das Berufsbild „Betriebsarzt“.

Im IfSG sind zwei Aussagen wegweisend: $\$ 3$ besagt, dass Information und Aufklärung der Allgemeinheit über die Gefahren übertragbarer Krankheiten und die Möglichkeiten zu deren Verhütung eine öffentliche Aufgabe sind. $\$ 1$ Abs. 2 S. 2 IfSG postuliert: „Die Eigenverantwortung der Träger und Leiter von Gemeinschaftseinrichtungen, Lebensmit- telbetrieben sowie des Einzelnen bei der Prävention übertragbarer Krankheiten soll verdeutlicht und gefördert werden."

\section{„Bei Maßnahmen des Infektionsschutzes sollte - wenn möglich - Prävention durch Information und Aufklärung erfolgen."}

Nun darf die Bedeutung von Generalklauseln (und als solche sind die beiden zitierten Vorschriften zu sehen) nicht zu sehr strapaziert werden. Der Vorwurf einer Überinterpretation kann aber sicherlich nicht erhoben werden, wenn diese Bestimmungen als Grundsatzentscheidung für die Anwendung und Auslegung der speziellen Regelungen im IfSG herangezogen werden. In der amtlichen Begründung zum Gesetzentwurf der Bundesregierung wird dazu ausgeführt: „Ein wichtiger Zweck des Gesetzes ist es, die Regeln für eine organisierte, effektive und vertrauensvolle Zusammenarbeit festzulegen“ [2].

Fasst man diese Gedanken zusammen, ist im Ergebnis gewollt, dass bei Maßnahmen des Infektionsschutzes wann immer möglich - Prävention durch Information und Aufklärung erfolgen soll. Verpflichtet wird ausdrücklich der öffentliche Gesundheitsdienst. Dies entbindet jedoch öffentliche und private Einrichtungen sowie Unternehmer nicht, eigenverantwortlich über den Schutz vor Übertragung von Infektionskrankheiten zu informieren. Ein einheitliches Konzept über Inhalte soll durch vertrauensvolle Zusammenarbeit öffentlicher und privater Stellen ermöglicht werden.

\section{Vorschriften für Schulen und sonstige Gemeinschafts- einrichtungen}

Die $\$ \$ 45$ bis 48 BSeuchG haben bisher den Infektionsschutz in Schulen und sonstigen Gemeinschaftseinrichtungen geregelt und diese Vorschriften sahen ein Besuchsverbot bei bestimmten übertragbaren Krankheiten vor, zu denen u. a. Pocken, Tularämie, Ornithose und Virushepatiden ohne eine Differenzierung gehörten. Erkrankten Beschäftigte oder Besucher von Gemeinschaftseinrichtungen oder schieden diese die Krankheitserreger aus, statuierte $\$ 45$
BSeuchG ein Besuchsverbot, bis nach dem Urteil des behandelnden Arztes oder des Gesundheitsamtes, eine Weiterverbreitung der Erkrankung nicht mehr zu befürchten war. Dieses Verfahren ist durch $\$ 34$ IfSG prinzipiell übernommen worden. Allerdings gibt es Änderungen, die im Detail für die Praxis bedeutende Auswirkungen haben.

Der Katalog der Erkrankungen, die im Einzelfall zum Besuchs- oder Tätigkeitsverbot führen, wurde dem heutigen Kenntnisstand und der epidemiologischen Situation angepasst. Genannt sind Erkrankungen, die im Einzelfall schwer verlaufen oder für deren Ausbreitung in Gemeinschaftseinrichtungen besonders günstige Bedingungen vorliegen. Außerdem ist der Läusebefall genannt. Kinder mit Gastroenteritis, die jünger als sechs Jahre sind, dürfen Gemeinschaftseinrichtungen ebenfalls nicht besuchen (Übersicht 1).

Festzuhalten ist, dass im Gesetzestext das Gesundheitsamt als Behörde, die eine Wiederzulassung zum Besuch feststellt, nicht mehr eigens genannt ist, sondern dies jetzt allein dem behandelnden Arzt übertragen ist.

Ausscheider von Choleravibrionen, Typhus- und Paratyphussalmonellen, Shigellen, EHEC und Diphtheriebakterien dürfen allerdings nur mit Zustimmung des Gesundheitsamtes und unter Beachtung von Schutzmaßnahmen die Einrichtung besuchen ( $\$ 34$ Abs. 2 IfSG).

Auch bestimmte Erkrankungen in der häuslichen Gemeinschaft lösen bei Personal oder Besuchern einer Gemeinschaftseinrichtung ein Besuchsverbot aus (Übersicht 2).

Liegen Umstände vor, wie in $\$ 34$ Abs. 1 bis 3 IfSG beschrieben, verlangen die Abs. 5 und 6 folgendes Verfahren: Die Gemeinschaftseinrichtung ist über Erkrankungen $\mathrm{zu}$ informieren und diese wiederum setzt davon das Gesundheitsamt in Kenntnis, damit ggf. besondere Schutzmaßnahmen veranlasst werden können.

Möglich wird diese Information aber nur, wenn Erkrankte oder deren Sorgeberechtigte Kindergemeinschaftseinrichtungen (siehe $\$ 33$ IfSG) über die im Gesetz genannten Erkrankungen auch informieren. Dies setzt eine Belehrung der potentiell Betroffenen voraus. Deshalb bestimmt $\$ 34$ Abs. 5 S. 2 IfSG, dass die Leitung der Gemeinschaftseinrichtung, jede Person, die dort neu be- 
treut wird oder deren Sorgeberechtigte, über die geschilderten Verpflichtungen aus $\$ 34$ Abs. 1 bis 3 IfSG zu belehren hat [3]. Für Betreuungs-, Erziehungs- und Aufsichtspersonal schreibt der $\$ 35$ IfSG vor, dass es vor erstmaliger Aufnahme der Tätigkeit und im Weiteren mindestens im Abstand von zwei Jahren über die gesundheitlichen Anforderungen und Mitwirkungspflichten nach $\$ 34$ IfSG zu belehren ist.

Im Gesetzgebungsverfahren wurde immer wieder Kritik geäußert, dem Gesundheitsamt würden durch die Neuregelung wichtige Regelungskompetenzen genommen. Dies trifft schon deshalb nicht zu, weil die $\$ \$ 33$ ff IfSG als ,besondere Vorschriften " benannt sind, neben denen die allgemeinen Regelungen über Verhütung und Bekämpfung übertragbarer Krankheiten ( $\$ 16$ oder 25 - 31 IfSG) uneingeschränkt Anwendung finden.

Entfallen ist die Untersuchung gem. $\S 47$ BSeuchG. Diese Leistungen des Gesundheitsamtes waren gebührenpflichtig und damit eine Einnahmequelle. Allerdings waren sie schon in der Vergangenheit aus Gründen der Infektionsprävention und unter epidemiologischen Gesichtspunkten eigentlich nicht mehr angezeigt [4].

Anstelle dieser Untersuchung tritt jetzt die Pflicht zur Belehrung der Beschäftigten (soweit sie Kontakt zu Kindern und Jugendlichen haben) und der Betreuten in Gemeinschaftseinrichtungen bzw. deren Sorgeberechtigten. Die Verletzungen der Pflichten aus $\$ \$ 34$ Abs. 1 und 3 sowie $\$ 35$ IfSG sind strafbzw. bußgeldbewehrt [5]. Um die Adressaten dieser Verpflichtungen in den Stand zu versetzen, den hier niedergelegten Sorgfaltspflichten nachzukommen, hat der Gesetzgeber Art und Umfang der Belehrung derart ausgestaltet, dass die Bedeutung und Gefahren von Infektionskrankheiten in den beiden Zielgruppen auch tatsächlich verstanden werden. Hierbei trifft Lehrer, Erzieher und andere Betreuer im Rahmen ihrer Aufsicht eine höhere Verantwortlichkeit als Besucher und deren Eltern. Letztere sind nur einmal vor erstmaligem Besuch einer Gemeinschaftseinrichtung zu belehren. Das Wissen des Personals hingegen muss spätestens alle zwei Jahre aufgefrischt werden.

Damit wird nachvollziehbar belegt, dass die eingangs beschriebenen Leitgedanken des Gesetzes

\section{Übersicht 1: \\ §34 Abs. 1 IfSG Gesundheitliche Anforderungen, Mitwirkungspflichten, Aufgaben des Gesundheitsamtes}

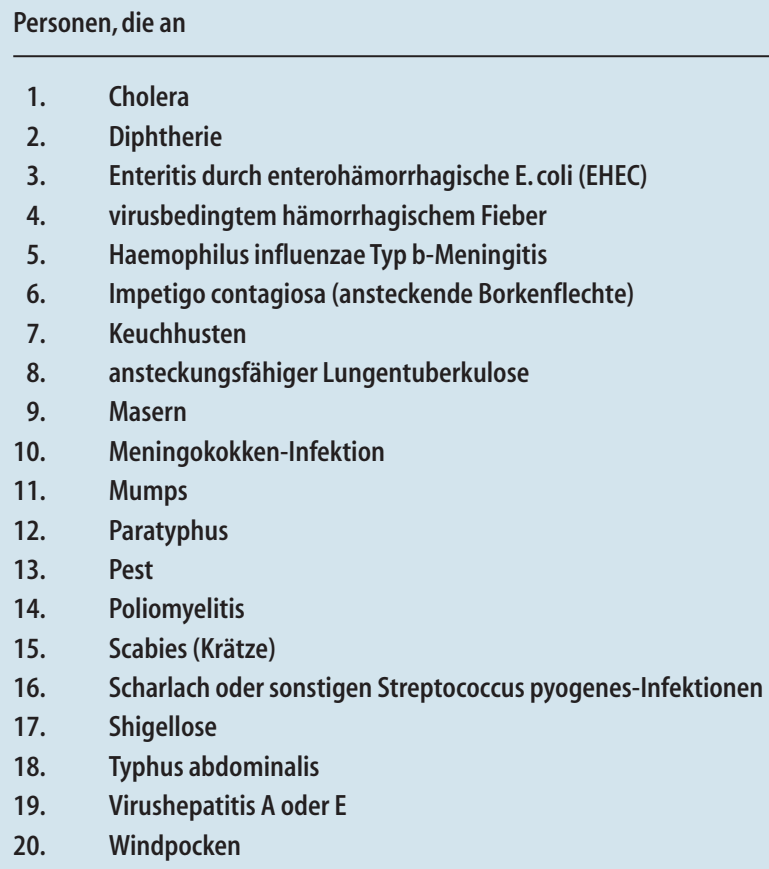

erkrankt oder dessen verdächtig oder die verlaust sind, dürfen in den in § 33 genannten Gemeinschaftseinrichtungen keine Lehr-, Erziehungs-, Pflege-, Aufsichts- oder sonstige Tätigkeiten ausüben, bei denen sie Kontakt zu den dort Betreuten haben, bis nach ärztlichem Urteil eine Weiterverbreitung der Krankheit oder der Verlausung durch sie nicht mehr zu befürchten ist. Satz 1 gilt entsprechend für die in der Gemeinschaftseinrichtung Betreuten mit der Maßgabe, dass sie die dem Betrieb der Gemeinschaftseinrichtung dienenden Räume nicht betreten, Einrichtungen der Gemeinschaftseinrichtung nicht benutzen und an Veranstaltungen der Gemeinschaftseinrichtung nicht teilnehmen dürfen. Satz 2 gilt auch für Kinder, die das 6. Lebensjahr noch nicht vollendet haben und an infektiöser Gastroenteritis erkrankt oder dessen verdächtig sind.

D Prävention durch Information und Aufklärung und

D Eigenverantwortung von Gemeinschaftseinrichtungen

konsequent durch Inhalt und Verfahren $\operatorname{der} \$ \$ 33$ ff IfSG umgesetzt sind.

\section{„Neben einer mündlichen Belehrung ist eine zielgruppen- spezifische Erläuterung mittels Merkblättern zweckmäßig."}

Dass gegen die Praktikabilität Bedenken vorgetragen werden, ist verständlich und doch ist noch einmal zu betonen, dass Kontroll- und Schutzmaßnahmen durch den öffentlichen Gesundheits- dienst in der Substanz gegenüber der alten Rechtslage unverändert geblieben sind.Vielmehr wurde eine Verbesserung dadurch erreicht, dass jetzt durch $\$ 34$ Abs. 6 IfSG eine förmliche Pflicht zur Information des Gesundheitsamtes durch die Einrichtung vorgesehen ist, wenn Infektionskrankheiten, die in $\$ 34$ Abs. 1 IfSG genannt sind, bei Personal oder Betreuten bekannt werden. Das erforderliche Basiswissen wird durch die Belehrung vermittelt.

Über die Form der Belehrung werden im IfSG keine Festlegungen getroffen. Allerdings ist neben einer mündlichen Belehrung eine zielgruppenspezifische Erläuterung mittels eines Merkblattes zweckmäßig [3] und angesichts der großen Zahl ausländischer Eltern, 
Übersicht 2:

\section{$\S 34$ Abs. 3 IfSG}

(3) Absatz 1 Satz 1 und 2 gilt entsprechend für Personen, in deren Wohngemeinschaft nach ärztlichem Urteil eine Erkrankung an oder ein Verdacht auf

1. Cholera
2. $\quad$ Diphterie
3. $\quad$ Enteritis durch enterohämorrhagische E. coli (EHEC)
4. $\quad$ virusbedingtem hämorrhagischem Fieber
5. Haemophilus influenzae Typ b-Meningitis
6. $\quad$ ansteckungsfähiger Lungentuberkulose
7. Masern
8. Meningokokken-Infektion
9. Mumps
10. Paratyphus
11. Pest
12. Poliomyelitis
13. Shigellose
14. Typhus abdominalis
15. Virushepatitis A oder E
aufgetreten ist.

die der deutschen Sprache nur mangelhaft mächtig sind, wird in naher $\mathrm{Zu}-$ kunft die Übersetzung dieser Merkblätter eine vordringliche Aufgabe sein (das RKI hat den Ländern Übersetzungen in englisch, französisch, spanisch, russisch und türkisch zur Verfügung gestellt).

\section{Belehrung über Infektions- krankheiten}

$\$ 34$ Abs. 11 IfSG verlangt erstmalig in einem Bundesgesetz, dass vor Aufnahme in die erste Klasse einer allgemeinbildenden Schule das Gesundheitsamt den Impfstatus zu erheben hat. Dabei hatte der Gesetzgeber im Auge, dass durch die Schulgesetze der Länder eine ärztliche Untersuchung durch Gesundheitsämter vor Einschulung allgemein vorgesehen ist.

Weiter beinhalten in $\$ 34$ die Abs. 2, 6 und 7 Regelungen, wonach alle Informationen über Infektionskrankheiten in den Einrichtungen beim Gesundheitsamt zusammenlaufen und dieses die Leitungen bzw. die Träger in allen Fachfragen berät; für bestimmte Ausscheider liegt die Entscheidung über eine Wiederzulassung zum Besuch allein beim Gesundheitsamt. Damit wird deutlich, dass zwischen Einrichtungen und Gesundheitsämtern in Fragen des Infektionsschutzes zumindest anlassbezogene, häufig jedoch dauerhafte und enge Kontakte bestehen.
Dass die Belehrung gem. $\$ 34$ Abs. 5 S. 2 (für Jugendliche bzw. für die Sorgeberechtigten von Kindern) den Einrichtungen selbst übertragen ist, bedeutet nicht, dass sie diese auch durch eigenes Personal oder Bedienstete zu erbringen haben. Vielmehr steht es Schulen, Kindergärten, Tagesstätten oder Internaten frei, sich ärztlichen Sachverstandes zu bedienen. Deshalb liegt nahe, dass die oben angesprochene schriftliche Belehrung mit der Schuleingangsuntersuchung durch Fachpersonal des Gesundheitsamtes übergeben und ggf. mündlich erläutert wird.

Von größerer Bedeutung ist wegen der im Gesetz vorgesehenen Wiederholung alle zwei Jahre die in $\$ 35$ IfSG beschriebene Belehrung für Beschäftigte in Gemeinschaftseinrichtungen, die nach dem Konzept des RKI Lehrer, Erzieher, Betreuer u. a. Personal, das Kontakt zu Kindern und Jugendlichen hat, über

D klinisches Bild,

- Übertragungswege,

D Inkubationszeit und

D Therapie (inkl. Impfprophylaxe)

der in $\$ 34$ IfSG genannten Erkrankungen informieren will [3].

Auch wenn über die Art der Belehrung bisher kaum praktische Erfahrungen vorliegen, gehen die Kommentatoren zum IfSG davon aus, dass eine mündliche Belehrung mit anschließender Aushändigung eines Merkblattes die geeignete Form ist [6]. Die Vermittlung medizinischer Inhalte zum Krankheitsbild Diphtherie, virusbedingte hämorrhagische Fieber, hämolytisch-urämisches Syndrom, Meningokokken-Infektion, Typhus u. a. kann in einem Gymnasium möglicherweise auch durch einen medizinisch interessierten Biologielehrer erfolgen. Die meisten Einrichtungen werden dazu - auch wegen auftauchender Fachfragen - nicht in der Lage sein.

Es wird also Arbeitgebern und Dienstherren obliegen, die geschilderten Belehrungen zu organisieren und sich qualifizierten medizinischen Sachverstandes zu bedienen.

Dass dies das Gesundheitsamt als kommunale Gesundheitsfachbehörde sein kann, ist gerade bei staatlichen Einrichtungen naheliegend. So hat z. B. das Sozialministerium Baden-Württemberg den Gesundheitsämtern mit Erlass vom 13. Dez. 2000 Merkblätter für die Belehrung in Schulen und sonstigen Gemeinschaftseinrichtungen zur Verfügung gestellt.

Die im Gesetz postulierte gegenseitige Unterstützung kann im konkreten Fall aber auch bedeuten, dass Betriebsärzte Belehrungen übernehmen. Sie sind in den Einrichtungen bekannt, mit den örtlichen Besonderheiten vertraut und die Vermittlung von fachlichen Inhalten gehört zu ihren Aufgaben.

\section{Belehrung für Beschäftigte in Lebensmittelbetrieben}

Anstelle der bisher durchgeführten Untersuchungen gem. $\$ 18$ Abs. 1 BSeuch sieht das IfSG vor, dass Personen, die gewerbsmäßigen Umgang mit bestimmten Lebensmitteln ( $\$ 42$ Abs. 2 IfSG) haben und dabei mit ihnen direkt oder indirekt (über Bedarfsgegenstände) in Berührung kommen oder in Küchen von Gaststätten, Restaurants, Kantinen, Cafés oder in sonstigen Einrichtungen mit und zur Gemeinschaftsverpflegung tätig sind, durch Belehrungen in die Lage versetzt werden, Hinderungsgründe bei sich selbst festzustellen. Für die erste Belehrung durch das Gesundheitsamt oder einen durch ihn beauftragten Arzt besagt $\$$ 43 Abs.1 IfSG, dass diese in mündlicher und schriftlicher Form erfolgen muss.

Eine häufig gestellte Frage ist, ob die Inhaber eines Zeugnisses gem. $\$ 18 \mathrm{Abs} .1$ (bisheriger „Lebensmittelausweis“) eine 
neue Bescheinigung nach IfSG beantragen müssen. Zur Beantwortung hilft ein Blick in $\$ 77$ Abs. 2 IfSG, wonach ein Zeugnis nach $\$ 18$ BSeuchG als Bescheinigung nach $₫ 43$ Abs. 1 IfSG (weiter) gilt. Allerdings wird für die Teilnahme an den jährlichen Belehrungen über Tätigkeitsverbote und Informationspflichten von Beschäftigten nicht zwischen „Alterlaubnissen“ und „Neubescheinigungen “ differenziert. Hier kommt es allein auf die ausgeübte Tätigkeit an (s. o. $\$ 42$ Abs. 1 IfSG) und alle Beschäftigten müssen teilnehmen.

$\$ 18$ Abs. 4 BSeuchG sah vor, dass die seinerzeit notwendigen Zeugnisse auch durch einen Arzt außerhalb des Gesundheitsamtes ausgestellt werden konnten, wenn dieser über die für die Untersuchungen notwendigen Einrichtungen verfügte. Dazu war eine Zulassung, also ein begünstigender Verwaltungsakt erforderlich. Diese Regelung ist in $\$ 43$ IfSG nicht mehr enthalten, da Stuhluntersuchungen und solche auf Tuberkulose nicht mehr routinemäßig stattfinden. Die Vorschrift führt aber an, dass ein vom Gesundheitsamt beauftragter Arzt die jetzt erforderlichen Belehrungen durchführen und die notwendigen Zeugnisse ausstellen kann. Aus dieser grundsätzlichen Neuregelung wird zu Recht gefolgert, dass es zur Beauftragung eines Arztes eines erneuten Verfahrens bedarf [7].

Es liegt nahe, dass Betriebsärzte im Rahmen von Einstellungsuntersuchungen oder durch die arbeitsmedizinische Betreuung von (Groß)betrieben zur Entlastung von Gesundheitsämtern beitragen können.

Die Dimension der Aufgabe wird deutlich, wenn man sich vor Augen hält, dass z. B. im Bezirk Charlottenburg (von Berlin) 1999 rund 8650 Lebensmittelzeugnisse ausgestellt wurden; in Frankfurt am Main waren es im gleichen Jahr ca. 6600. $\mathrm{Da}$ es sich bei der Bescheinigung gem. $\$ 43$ Abs. 1 IfSG und dem früheren Zeugnis gem. $\$ 18$ Abs. 1 BSeuchG um Aufgaben handelt, die - mit veränderten Inhalten qua Gesetz den Gesundheitsämtern übertragen sind, fanden sich schon zu Jahresbeginn im Informationsverbund Umwelt und Gesundheit (UmInfo), dem ÖGD-Intranet [8], zahlreiche Hinweise zur Organisation der Belehrungen und dazu, wie die mündliche Belehrung visuell unterstützt werden kann.

Im Vorfeld war als größtes Problem die fremdsprachliche Übersetzung der schriftlichen Belehrungsmaterialien angesehen worden, da gerade im Gaststättengewerbe und bei Cateringunterneh- men ein Großteil der Beschäftigten Ausländer mit z. T. mangelhaften Deutschkenntnissen sind. Umso erfreulicher ist, dass durch Eigeninitiative von Gesundheitsämtern und anderen Stellen des ÖGD Produkte für den mündlichen Teil der Belehrung entstanden sind, die mit Symbolen und bewegten Bildern arbeiten und zum Verständnis nur Grundkenntnisse in Deutsch verlangen [9]. Dass auch diese Entscheidung für eine Prävention durch Information und Aufklärung nicht nur im Kontext des IfSG sondern auch im größeren Zusammenhang des Verbraucherschutzes richtig ist, belegt ein Blick in die Lebensmittelhygiene-Verordnung (LMHV). Auch dort wird in $\$ 4$ Abs. 2 von Arbeitgebern verlangt, dass sie durch betriebseigene Maßnahmen zu gewährleisten haben, dass Personen, die mit Lebensmitteln umgehen, entsprechend ihrer Tätigkeit und unter Berücksichtigung ihrer Ausbildung in Fragen der Lebensmittelhygiene unterrichtet und geschult werden. Zwar werden in der Anlage zur VO, Kapitel 5 Ziffer 7 auch Aussagen über die Einhaltung der Personalhygiene gemacht; Schwerpunkte der Vorschrift sind aber Regelungen zur Produkthygiene.

\section{„Es bietet sich an, Unterrichts- einheiten gemäß Lebensmit- tel-Hygieneverordnung und Infektionsschutzgesetz zusammenzufassen und inhaltlich zu verknüpfen."}

Kritiker haben deshalb von Doppelregelung gesprochen bzw. waren der Auffassung, die gesetzliche Fixierung auch dieser Belehrung sei besser in einem Regelwerk des Lebensmittelrechts untergebracht worden [10]. Dem ist entgegenzuhalten, dass die Inhalte der Belehrung gem. IfSG humanmedizinische Inhalte betreffen, die qua Fachkompetenz von Gesundheitsämtern und Betriebsärzten am besten dargestellt werden können. Inhalt der Belehrung ist die Vermittlung von Wissen über Enteritiden und andere Erkrankungen, deren Erreger in Lebensmitteln Verbraucher nach Genuss krank machen können (z. B. Typhus, Hepatitis A, EHEC und Staphylokokken).

Diese Trennung von medizinischen Inhalten in der Schulung von Beschäftigten zu den Aspekten der Produkthy- giene erzeugt keinen unkoordinierten und unnötigen Wust von Belehrungen, sondern es sollte möglich sein, die Unterrichtseinheiten gem. LMHV und IfSG zusammenzufassen und inhaltlich $\mathrm{zu}$ verknüpfen. Gerade weil die im IfSG genannten Erkrankungen bei uns nur selten und in manchen Heimat- und Urlaubsländern von Beschäftigten häufiger sein können, ist eine Terminierung der jährlichen IfSG-Belehrung vor der Urlaubszeit sinnvoll und sollte Anlass sein, über Krankheitszeichen, Epidemiologie, Übertragungswege, Inkubationszeiten und Therapie (einschließlich einer möglichen Schutzimpfung) von

- Typhus abdominalis,

- Paratyphus,

- Cholera,

- Shigellenruhr,

- Salmonellose,

- anderen infektiösen Gastroenteriden,

- Virushepatits A oder E,

- infizierten Wunden,

- anderen Hauterkrankungen mit Krankheitserregern, die über Lebensmittel übertragen werden können

$\mathrm{zu}$ informieren. (Aufgeführt ist der gesamte und abschließende Katalog aus $₫$ 42 Abs.1 Nr.1 und 2. Nr. 3 der Vorschrift beschreibt außerdem, dass ein Hinderungsgrund für die Tätigkeit auch die Ausscheidung von Shigellen, Salmonellen, EHEC und Choleravibrionen ist.)

\section{Wegfall der Stuhluntersuchungen}

Gem. $§ 18$ BSeuchG war die Ausstellung des Zeugnisses an zwei negative Stuhlproben und den Ausschluss einer Lungentuberkulose geknüpft. Der Wegfall dieser Untersuchungen wurde von Vertretern aus dem ÖGD z. T. heftig kritisiert, weil so Ausscheider nicht mehr identifiziert werden könnten.

Da die gesetzlichen Anforderungen früher nur eine Untersuchung vor erstmaliger Aufnahme der Tätigkeit verlangten und danach nie wieder, gleichgültig, ob die Tätigkeit lange Zeit unterbrochen wurde oder nicht, ist unstrittig, dass die Verfahrensweise nicht geeignet war, Epidemien durch kontaminierte Lebensmittel zu vermeiden. Schon 1979 wurde in der amtlichen Begründung zur 
damaligen Novelle zum BSeuchG ausgeführt: „Zwar findet man bei Untersuchungen nach einem Ausbruch von Salmonellenerkrankungen oft unter dem Personal des Lebensmittelbetriebes oder der Küche von denen die Erkrankungen ausgingen, einen Ausscheider. Nur bleibt offen, ob er wirklich das Lebensmittel kontaminiert hat oder ob er nicht vielmehr ebenso Opfer der auf andere Weise kontaminierten Lebensmittel ist wie die anderen Erkrankten. Für letzteres gibt es einige Anhaltspunkte, für ersteres dagegen nicht" [11].

Tatsächlich lag in den goer Jahren die Prävalenz positiver Stuhlproben auf Salmonellen in mehreren Untersuchungen in Deutschland zwischen 0,3 und $0,7 \%$ für Beschäftigte in Lebensmittelbetrieben. Ein Zusammenhang zwischen Untersuchungspraxis und infektionsepidemiologischen Erkenntnissen über nahrungsmittelbedingte Infektionen war nicht zu erkennen [12].

\section{"Stuhluntersuchungen stellen eine Momentaufnahme dar und vermitteln eine falsche Sicherheit."}

Einmalige oder auch jährliche Stuhluntersuchungen stellen eine Momentaufnahme dar und vermitteln auch angesichts der Tatsache, dass oft Krankheitserreger nur intermittierend ausgeschieden werden, eine falsche Sicherheit.

$\mathrm{Zu}$ bedenken ist auch, dass anlassbezogene Untersuchungen und Maßnahmen jederzeit aufgrund der $\$ \$ 16$ oder 25 bis 31 IfSG angeordnet werden können, wenn es in einer Kindergemeinschaftseinrichtung, aber auch in einem Altenheim oder einem Krankenhaus zu einer Häufung von Gastroenteriden gekommen ist.

\section{Durchführung von Bundesgesetzen}

Die meisten Anfragen an das RKI betreffen den Kreis der im Gesetz nicht eindeutig genannten Adressaten von Belehrungen in Gemeinschaftseinrichtungen und Personen, die nicht gewerbsmäßig Le- bensmittel herstellen oder inverkehrbringen. So ging es beispielsweise um das Problem, ob das Reinigungspersonal in Schulen oder das Pflegepersonal in Altenheimen, das Brote schmiert und kleine Speisen zubereitet, auch gem. IfSG zu belehren seien. In diesen beiden Fällen war die Antwort relativ einfach. Das Reinigungspersonal kommt mit den Betreuten normalerweise nicht in Kontakt $(\$ 34$ Abs. 1 IfSG). Pflegepersonal bedurfte früher keines Lebensmittelausweises gem. $\$$ 18 BSeuchG, so dass bei einer Bewertung weniger die Auslegung einzelner Rechtsbegriffe als vielmehr die bisher geübte Praxis unter Berücksichtigung des Gefährdungspotentials weiterhelfen kann. An den Beispielen wird aber auch deutlich, wo die Grenzen der Stellungnahmen aus dem RKI erreicht sind.

Gem. Art. 83 GG führen die Länder die Bundesgesetze als eigene Angelegenheit aus. Das bedeutet, dass auch die Festlegung des Verfahrens, Art der Belehrung und weitere Konkretisierung der Vorschriften für Gemeinschaftseinrichtungen und Lebensmittelbetriebe den zuständigen Stellen in den Ländern obliegt und eine für die Anwender verbindliche Auslegung von Rechtsbegriffen regelmäßig durch Erlasse der Obersten Landesgesundheitsbehörde und Verfügungen der nachgeordneten $\mathrm{Be}$ hörden erfolgt.

\section{Schlussbemerkung}

Obwohl das IfSG am 20. Juli 2000 im Bundesgesetzblatt verkündet wurde und im öffentlichen Gesundheitsdienst intensiv und zeitnah organisatorische Vorbereitungen zur Umsetzung des Gesetzes getroffen wurden, stehen auch für die Zukunft noch weitere Entscheidungen und Verfahrensregeln an, die im Einzelfall schwierig sein können. Insbesondere Gesundheitsämter hatten aus den $\$ \$ 18$ und 47 BSeuchG Gebühreneinnahmen, die jetzt bzgl. der Beschäftigten in Gemeinschaftseinrichtungen (keine Untersuchungen mehr) entfallen. Andererseits haben in beiden Bereichen durch die Wiederholungsbelehrungen in den Einrichtungen und Unternehmen die Aufgaben, bei denen medizinischer Sachverstand gefordert ist, erheblich zugenommen. Bei Absprachen über Arbeitsteilung zwischen Gesund- heitsämtern und Betriebsärzten gerade bei den Wiederholungsbelehrungen sollte es weniger um Wettbewerb mit zu unterbietenden Gebührenrahmen gehen, als um Sicherstellung von Qualität der Unterrichtung.

\section{Literatur}

1. Nassauer A, Mielke M (2000) Rechtsgrundlagen zum Infektionsschutz im Krankenhaus. Bundesgesundheitsbl 43:459-465

2. Deutscher Bundestag, 4. Wahlperiode (2000) Gesetzentwurf der Bundesregierung zum Seuchenrechtsneuordnungsgesetz. DS 14/2530:43

3. Ein Vorschlag des RKI für entsprechende Merkblätter an die obersten Landesgesundheitsbehörden ist im Internet unter www.rki.de/INFEKT/IFSG/IFSG.HTM verfügbar

4. Deutscher Bundestag, 4. Wahlperiode (2000) Gesetzentwurf der Bundesregierung zum Seuchenrechtsneuordnungsgesetz. DS 14/2530:78

5. Zur weiteren Darstellung siehe: Bales $S$, Baumann HG (2001) Infektionsschutzgesetz, $\S 34$ Rn. 25, Köln Kommentar.W. Kohlhammer

6. Bales S, Baumann HG (2000)Infektionsschutzgesetz, §34 Rn. 16, Köln Kommentar. W. Kohlhammer

7. Z.B.: Erlass des Sozialministeriums Baden-Württemberg vom 13.Dez.2000, Az.: 52-5420.11-2

8. Diese System wird vom RKI betreut und gepflegt. Ansprechpartner ist Herr Dr. U. Kaiser, Tel.: 01888-754-3197. Der Zugang ist nur nach Vergabe einer Benutzer-ID und eines Passwortes möglich

9. Zu empfehlen ist eine $C D$, die vom RP Kassel und den Gesundheitsämtern Kassel Stadt und Landkreis produziert wurde. Copyright: Hans-Martin Merkwirth, c/o Gesundheitsamt Landkreis Kassel, Humboldtstr. 22-26,34117 Kassel

10. NN (2000) Mehr Pflichten durch ein neues Infektionsschutzgesetz. GV-Kompakt 11:50-52

11. Schumacher W, Meyn E (1992) Bundes-Seuchengesetz (Kommentar), 4. Aufl. Deutscher Gemeindeverlag, Köln, S 78

12. Heudorf U (1998) Zur Diskussion um Voraussetzungen für Lebensmittelzeugnisse des Gesundheitsamtes - $§ 18$ Bundes-Seuchengesetz und $\S 43$ Infektionsschutzgesetz (Entwurf).Gesundheitswesen 60:166-169 